
РЕЗИСТЕНТНЫХ ПРОЛАКТИНОМ: КЛИНИЧЕСКОЕ НАБЛЮДЕНИЕ

\author{
( ) З.А. Калмыкова*, С.Ю. Воротникова, Н.С.Федорова, Л.К.Дзеранова, Е.А.Пигарова, А.В.Воронцов
}

ФГБУ «Национальный медицинский исследовательский центр эндокринологии» Минздрава России, Москва, Россия

Гиперпролактинемия (ГП) - одно из наиболее распространенных нейроэндокринных нарушений. В 60\% случаев патологическая ГП обусловлена наличием пролактин-секретирующей аденомы гипофиза. Терапия агонистами дофаминовых рецепторов 2 типа (АД2р) - метод выбора для лечения патологической ГП, позволяющий добиться нормализации уровня ПРЛ и уменьшения размеров аденомы гипофиза в большинстве случаев. Однако, у 15-20\% пациентов наблюдается резистентность к терапии АД2р и вопрос её преодоления остается открытым. Рассматриваются различные подходы к решению данного вопроса, основным из которых является увеличение дозы АД2р до максимально переносимой. В данной статье мы представляем клиническое наблюдение пациента с частичной резистентностью к АД2р, демонстрирующее хороший ответ на лечение высокими дозами каберголина.

КЛЮЧЕВЫЕ СЛОВА: гиперпролактинемия; пролактинома; агонисты дофаминовых рецепторов; каберголин; резистентность.

\title{
THE EFFICACY OF HIGH-DOSE CABERGOLINE TREATMENT OF PROLACTINOMAS RESISTANT TO STANDARD DOSES: A CLINICAL OBSERVATION
}

(c) Zilya A. Kalmykova*, Svetlana Y. Vorotnikova, Natalia S. Fedorova, Larisa K. Dzeranova, Ekaterina A. Pigarova, Aleksandr V. Vorontsov

Endocrinology Research Centre, Moscow, Russia

Hyperprolactinemia (HP) is one of the most common neuroendocrine disorders. In $60 \%$ of cases, pathological HP is caused by pituitary prolactin-secreting adenoma. Therapy with agonists of dopamine type 2 receptors (D2 receptor agonists) is a method of choice for the treatment of pathological HP which allows to achieve prolactin normalization and reduction of pituitary adenoma in most cases. However, $15-20 \%$ of patients are resistant to D2 receptor agonists, and the question of overcoming this resistance is highly relevant. Different approaches are considered to solve this problem, one - is to increase the dose of D2 receptor agonists up to the maximally tolerated. In this article, we present a clinical observation of a patient with a partial resistance to $\mathrm{D} 2$ receptor agonists who demonstrated a good response to treatment with high doses of cabergoline.

KEYWORDS: hyperprolactinemia; prolactinoma; dopamine receptor agonists; cabergoline; resistance.

\section{АКТУАЛЬНОСТЬ}

Гиперпролактинемия (ГП) - одно из наиболее распространенных нарушений, связанных с патологией гипоталамо-гипофизарной системы [1,2]. Диагностика и лечение ГП сохраняет свою актуальность в клинической практике как эндокринологов, так и врачей других специальностей. ГП полиэтиологична и может наблюдаться при различных клинических и физиологических состояниях, а также при приеме некоторых лекарственных средств [3]. Многообразие клинических проявлений ГП определяется широким спектром биологического действия ПРЛ как у мужчин, так и у женщин: помимо хорошо изученного влияния на репродуктивную систему, ПРЛ оказывает действие на липидный и углеводный обмены, иммунную систему, костный метаболизм [4].

В 60\% случаев патологическая ГП обусловлена гиперпродукцией ПРЛ лактотрофными аденомами гипофиза - пролактиномами. Пролактиномы являются наиболее распространенными среди гормонально ак- тивных опухолей гипоталамо-гипофизарной области их доля среди всех новообразований гипофиза около 40\% [1]. Пролактиномы, как и другие аденомы гипофиза, классифицируют по размеру: микроаденомы (до 10 мм) и макроаденомы (более 10 мм) [3].

Методом выбора при лечении пролактином является медикаментозная терапия агонистами дофаминовых рецепторов 2 типа (АД2р) [5]. В большинстве случаев лечение позволяет добиться устранения клинических симптомов, нормализации уровня ПРЛ и уменьшения размеров аденомы, предупреждения развития метаболических нарушений. Согласно международным клиническим рекомендациям, наиболее предпочтительным препаратом среди АД2р является высокоафинный АД2р - каберголин, имеющий наибольшую эффективность в нормализации уровня ПРЛ и уменьшения размеров опухоли [5-8].Несмотря на успехи медикаментозной терапии, приблизительно у $15-20 \%$ пациентов наблюдается резистентность к консервативному лечению - полная или частичная [9-11]. К основным критериям резистентности к АДр 
относят отсутствие нормализации уровня ПРЛ сыворотки, уменьшение размеров аденомы гипофиза менее $50 \%$ от исходного и персистенция клинических проявлений $[4,9,11,12]$. На фоне продолжительного лечения возможно уменьшение размеров аденомы гипофиза на менее чем на $50 \%$ от исходных. В то же время на фоне длительного применения АДр уровень ПРЛ может значительно снизиться без достижения при этом референсных значений, но с улучшением клинической картины заболевания: у женщин - возобновления менструального цикла и даже наступления беременности, у мужчин - повышения потенции, улучшения показателей спермограммы. Некоторые пациенты могут давать обратную реакцию на лечение: нормализацию уровня ПРЛ без изменения размера опухоли, или, наоборот, уменьшение размера опухоли с незначительными изменениями уровня ПРЛ $[9,13]$. Ведение таких пациентов является сложной задачей для врача-эндокринолога в связи неоднозначной эффективностью альтернативных методов лечения резистентных пролактином, таких как оперативное лечение и лучевая терапия, и отсутствием четких подходов к решению данной проблемы. В данной статье мы представляем клиническое наблюдение пациента Д. 19 лет с частичной резистентностью к консервативному лечению.

\section{ОПИСАНИЕ СЛУЧАЯ}

Пациент Д., 1994 г.р. впервые обратился в ФГБУ «НМИЦ эндокринологии» Минздрава России в 2015 г. с жалобами на выраженные головные боли, слабость и снижение потенции.

Из анамнеза известно, что в 2013г. пациент обратился к неврологу и терапевту по месту жительства с вышепредставленными жалобами. При гормональном обследовании выявлено значимое повышение уровня пролактина крови до 81000 мME/л (референсный диапазон 53-360). Проведена МРТ головного мозга (ГМ): выявлено объемное образование гипоталамо-гипофизарной системы размерами 34×29×35 мм с пара -, инфра -, анте -, ретроселлярным распространением. Установлен диагноз пролактин-секретирующей макроаденомы гипофиза, инициирована терапия каберголином 0.5 мг в неделю с постоянным постепенным увеличением дозы препарата до 1.5 мг в неделю в течение 3-ех месяцев. На фоне лечения пациент отметил улучшения общего самочувствия, снижение частоты головных болей, повышение потенции, поэтому в дальнейшем принимал терапию нерегулярно, динамического обследования не проходил.

С апреля 2015 года отметил нарастание интенсивности головных болей, что и послужило поводом для амбулаторного обращения в ФГБУ «НМИЦ эндокринологии» Минздрава России, через полтора года от дебюта заболевания. Пациент отмечал улучшение общего самочувствия в виде снижения интенсивности и частоты головных болей.

При осмотре состояние удовлетворительное. Рост 167 см, вес 70 кг, ИМТ - 25 мг/м². Кожные покровы чистые, физиологической окраски. Молочные железы не увеличены, безболезненны. При надавливании выделений из сосков нет.
При обследовании по данным гормонального анализа крови ПРЛ от 02.04.2015г. - 13124.0 мМЕ/л (референсный диапазон 60-510), макроПРЛ - 101.59 мМЕ/л. Проведена МРТГМ:картинамакроаденомыгипофизассупра-,пара-, инфра -, анте -, ретроселлярным распространением, размерами 27×28×34 мм; в переднем отделе тесно прилежит к левому зрительному нерву. При офтальмологическом обследовании поля зрения в норме, хиазмального синдрома нет. Учитывая недостижение нормализации уровня ПРЛ и отрицательную динамику размеров образования, при отсутствии абсолютных показаний к нейрохирургическому лечению, принято решение лечение каберголином с увеличением дозы под контролем уровня ПРЛ и динамического МРТ ГМ. В течение последующих 6-ти месяцев пациент постоянно принимал каберголин с постепенным увеличением дозы препарата с 1.5 мг до 4.5 мг под контролем уровня ПРЛ ежемесячно. На этом фоне ПРЛ крови от 05.09.2015 г - 1534 мЕд/л, по данным контрольной МРТ макроаденома с пара -, инфра -, анте-, ретроселлярным распространением, отмечается положительная динамика: редукция размера образования до $20 \times 22 \times 32$ мм и его супраселлярного распространения. Таким образом, у пациента отмечен хороший ответ на медикаментозную терапию в виде снижения уровня ПРЛ, уменьшения размера образования, улучшения общего самочувствия, а также хорошая переносимость препарата, что стало основанием для принятия решения на консилиуме врачей о дальнейшем увеличении дозы каберголина выше максимально допустимой согласно аннотации препарата. Было рекомендовано динамическое наблюдение с плановым проведением эхокардиографического исследования.

В феврале 2016 г госпитализирован в ФГБУ «НМИЦ эндокринологии» Минздрава России для обследования и определения дальнейшей тактики. На фоне регулярного приема каберголина в дозе 6.5 мг в неделю достигнута нормализация уровня ПРЛ - 381.7 мЕд/л (референсный диапазон 60 - 510). Проведена МРТ ГМ: по сравнению с исследованием от 2015 г отмечена положительная динамика в виде уменьшения размера образования и характера его распространения: пара -, инфра -, ретроселлярный рост, размеры до 20×21×25 мм. Пациенту рекомендовано продолжить прием каберголина в прежней дозе под контролем лабораторных и инструментальных методов исследования.

В дальнейшем на фоне постоянного приема вышеуказанной дозировки каберголина сохранялась положительная динамика: поддержание уровня ПРЛ в пределах целевых значений (ПРЛ от 18.01.2017 301.3 мЕд/л (референсный диапазон 60- 510)), уменьшение размеров аденомы (рис.1), что позволило в январе 2017 рекомендовать начать постепенное снижение дозы препарата.К декабрю 2018 г пациент принимал каберголин в дозе 0.5 мг в неделю, уровень ПРЛ находился в пределах нормы - 256 мME/л, по данным МРТ отмечалось дальнейшее уменьшение размеров пролактиномы: инфра -, параселлярное распространение, размеры 12×20x18 мм, снижение ретроселлярного распространения (рис.2). При эхокардиографическом исследовании структурных и функциональных нарушений не выявлено. В настоящее время наблюдение за пациентом продолжается. 


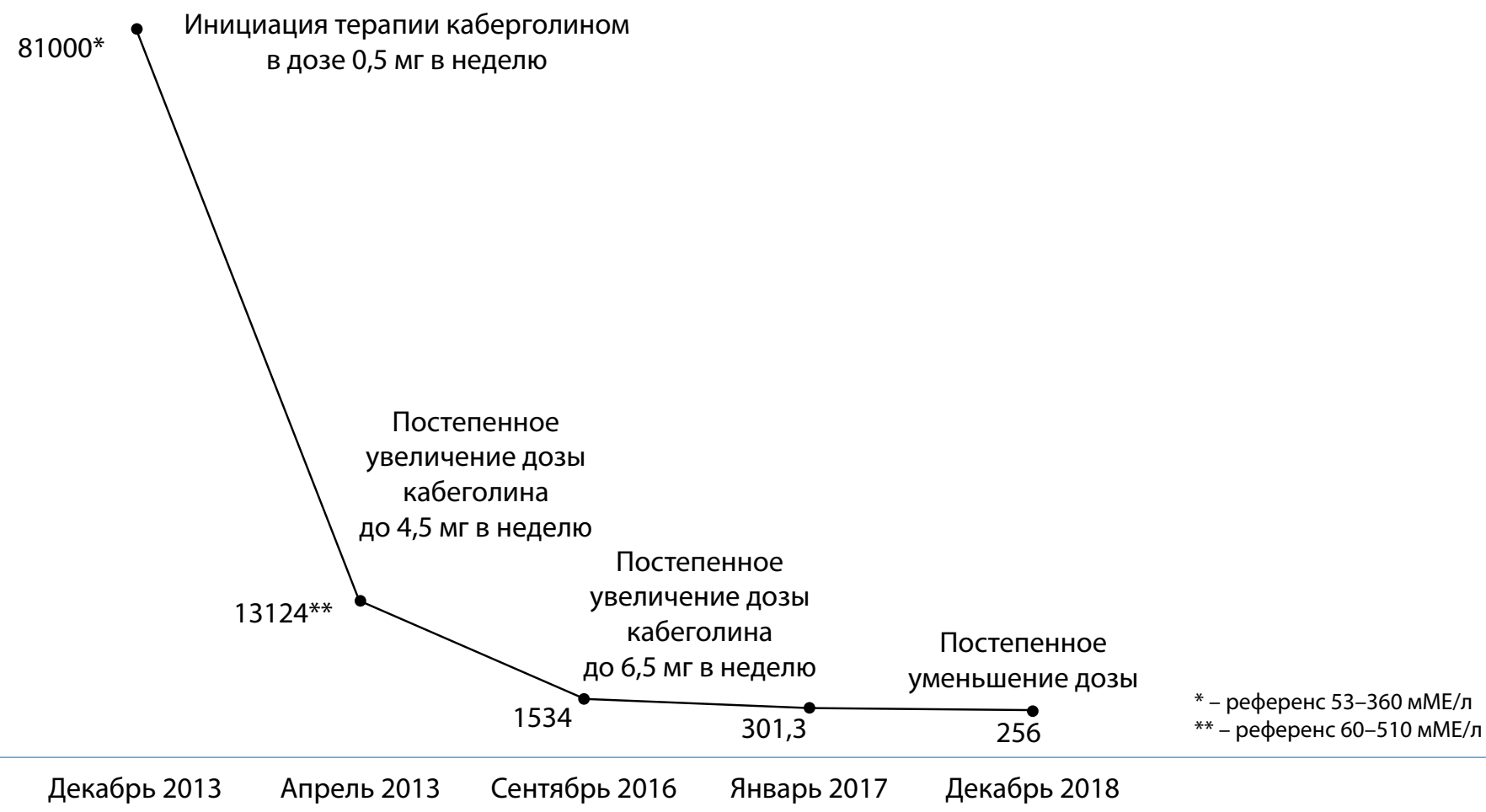

Рис. 1. Динамика изменения уровня пролактина в сыворотке крови на фоне терапии каберголином.

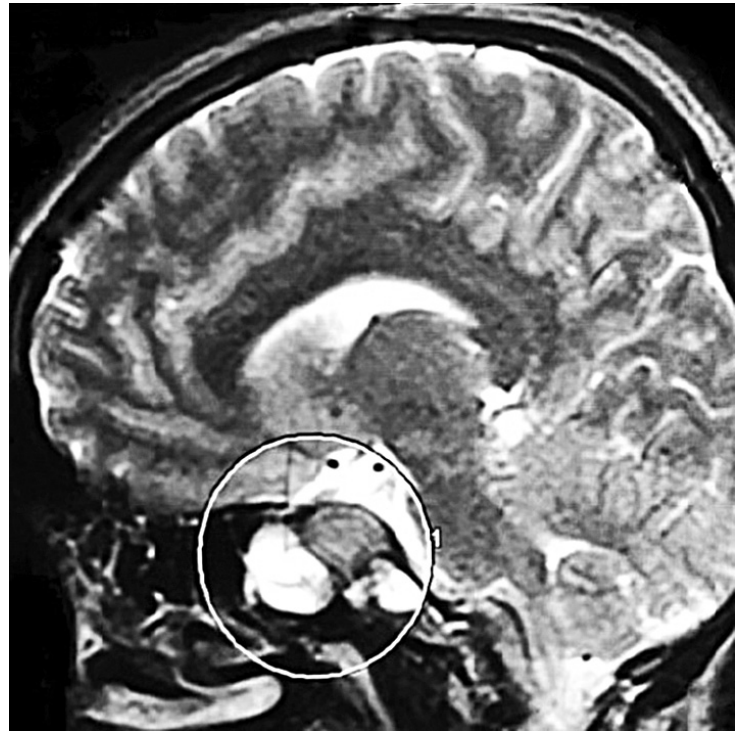

Декабрь 2013

$34 \times 29 \times 35 \mathrm{Mm}$

с пара -, инфра -, анте -,

ретроселлярным распространением

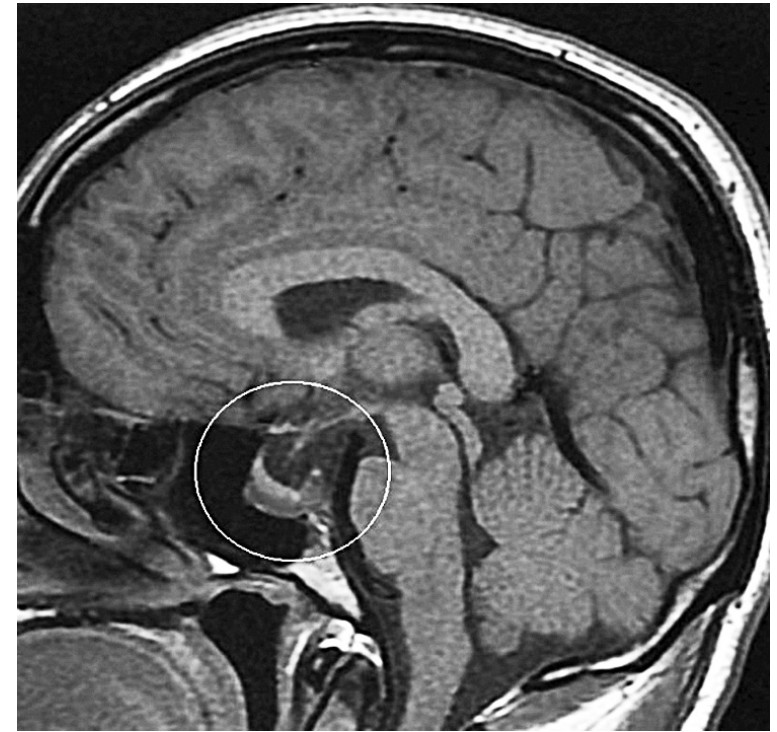

$12 \times 20 \times 18 \mathrm{MM}$

с пара- , инфраселлярным распространением

Рис. 2. Изменение размеров и распространения образования гипофиза через 5 лет терапии каберголином.

\section{ОБСУЖДЕНИЕ}

Вышеописанное клиническое наблюдение пациента с макропролактиномой и частичной резистентностью к терапии АД2р демонстрирует хороший клинический ответ при применении высоких доз каберголина, а также затрагивает вопрос возможных методов преодоления резистентности к АДр. В подобных случаях возможно несколько способов: замена одного препарата из группы АДр другим, увеличение дозы АДр, хирургическое лечение и лучевая терапия, а также назначение других препаратов, не относящихся к группе АДр [14] . В литературе имеются работы по применению аналогов соматостати- на, селективных модуляторов эстрогеновых рецепторов, темозоломида. Однако новые альтернативные фармакологические подходы к лечению таких пациентов требуют дальнейших исследований [9].

Учитывая данные о наибольшей эффективности каберголина по сравнению с другими препаратами данного класса, замена на другой препарат в данном случае не рассматривалась. Нейрохирургическое лечение не требовалось, так как абсолютных показаний к его проведению у пациента не было. На фоне постепенного увеличения дозы каберголина отмечено улучшение ответа, что позволило придерживаться данного метода лечения в вышеописанном случае. 
Вопрос возможности назначения высоких доз каберголина на сегодняшний день остается открытым [15]. Максимальная рекомендуемая доза препарата согласно его аннотации - 4.5 мг в неделю. Ограничения по максимальной дозировке связаны с возможной непереносимостью препарата и отсутствием доказательной базы по безопасности высоких доз. Непереносимость лекарственного средства проявляется совокупностью побочных эффектов, возникающих на фоне приема АДр в адекватной дозе. К наиболее распространённым побочным эффектам всех АДр относят головную боль, сонливость, головокружения, тошноту, рвоту, гипотонию $[2,16]$. В качестве одного из самых значимых осложнений АДр рассматривают развитие нарушений клапанного аппарата сердца, которые впервые были отмечены у пациентов с болезнью Паркинсона на фоне приема каберголина в дозах более 3 мг/сут $[17,18]$. Однако, опубликованные в 2008 г результаты исследования Molitch M. и соавт. показали безопасность данной группы препаратов в отношении клапанной патологии: среди 31829 исследуемых число новых случаев поражения клапанов сердца при лечении АД2р составило 2381 (9\%) среди пациентов с ГП, 23870 (75\%) в группе контроля, оперативное лечение понадобилось 2 пациентам (0.08\%), 28 (0.12\%) пациентам с ГП и без соответственно [19]. Описаны единичные случаи плевролегочных изменений на фоне высоких доз АДр при болезни Паркинсона, но согласно последним литературным данным, подобные реакции при данной патологии возможны и при лечении другими алкалоидами спорыньи [20,21]. Следует отметить, что все больше внимания уделяется возможности развития различных импульсивно-компульсивных расстройств (ИКР): игромании, гиперсексуальности, гиперфагии (патологическое переедание) и дофаминового дизрегуляционного синдрома, развитие которых, согласно ряду исследований ассоциировано с приемом высоких доз АДр [22]. Применение дофаминергических препаратов при различных патологиях (например, при болезни Паркинсона, при синдроме беспокойных ног и фибромиалгии) приводило к увеличению риска развития ИКР в 2-3.5 раза. В целом, подобные расстройства развиваются лишь у немногих лиц, получающих высокие дозы АДр, что может быть связано с влиянием генетических факторов, способствующих или, наоборот, препятствующих ИКР. Так, близнецовым методом было установлено, что роль генетических факторов в риске развития игромании составляет 33-54\% [22-25].

Накапливается все больше данных и об эффективности назначения высоких доз АД2р при резистентности к лечению. Проспективное исследование Ono M. и соавт, включало 150 пациентов с ГП, которых разделили на 3 группы: пациенты, не получавшие какую-либо терапию $(n=60)$, пациенты с непереносимостью других препаратов из группы АД2р, кроме каберголина $(n=64)$ и резистентные к терапии АД2р пациенты $(n=26)$. Всем пациентам инициирована терапия каберголином. Нормализация ПРЛ в первых двух группах отмечена суммарно у $83 \%$ через 3 месяца и у 95\% через 6 месяцев на фоне приема каберголина еженедельно в дозах $2.0 \pm 0.3$ мг в первой группе, $0.9 \pm 0.1$ мг - во второй. В группе резистентных пациентов этот показатель составил 35\% за 3 месяца и 58\% через 6 месяцев при применении $5.2 \pm$ 0.6 мг каберголина в неделю. В исследовании отмечена хорошая переносимость препарата, побочные эффекты выявлены только у 9\% пациентов [26]. Vilar и соавт. проспективно оценили течение ГП у 25 пациентов с пролактиномами и резистентностью к терапии каберголином в дозе 3 мг/нед при постепенном увеличении дозы препарата каждые 3 месяца, максимально до 9 мг/нед. Нормализация уровня ПРЛ была достигнута у 18 пациентов (72\%), из них: трех (12\%) на фоне приема 4 мг/нед; у девяти (36\%) - 5 мг/нед; и у шестерых (24\%) - 6-7 мг/ нед. Пациенты хорошо переносили препарат, при эхокардиографии данных за патологию клапанного аппарата не получено [20]. Описаны случаи, при которых положительный клинический эффект был достигнут лишь при повышении дозы каберголина до 21 мг/нед, побочные реакции при этом не развивались [28]. Однако, есть и исследования с обратными результатами. В работе Delgrange Е. и соавт. применение каберголина в дозах более 3.5 мг/нед не показало большего клинического эффекта в сравнении с приемом препарата в дозе менее 1.5 мг/нед [29]. Малая эффективность увеличения дозы более 3.0 мг отмечена и в другом ретроспективном исследовании [30]. В целом, имеющиеся литературные данные позволяют рассматривать назначение каберголина в дозах выше 4.5 мг/нед в качестве возможного подхода для пациентов с резистентностью к АД2р. Необходимо информировать пациентов о потенциальных побочных эффектах высоких доз каберголина и необходимости проходить регулярное эхокардиографическое исследование для оценки состояния клапанного аппарата.

\section{ЗАКЛЮЧЕНИЕ}

Данное клиническое наблюдение пациента с частичной резистентностью к АДр иллюстрирует возможность эффективного лечения пролактином высокими дозами каберголина. Таким образом, пациентам с частичной резистентностью к АДр может быть рекомендовано увеличение дозы каберголина до максимально переносимой, обращая при этом внимание на возможные побочные эффекты с целью их раннего выявления и предупреждения развития. Хирургическое лечение показано в случаях полной резистентности к терапии АДр и в острых ситуациях (ликворея, апоплексия гипофиза с развитием хиазмального синдрома). Частота резистентности к лечению АДр у пациентов с пролактиномами, особенно среди лиц репродуктивного возраста, а также отсутствие четких подходов к решению данной проблемы определяют необходимость ее дальнейшей разработки.

\section{ДОПОЛНИТЕЛЬНАЯ ИНФОРМАЦИЯ}

Источник финансирования. Подготовка и публикация рукописи проведены на личные средства авторского коллектива. Дополнительных источников финансирования не было.

Согласие пациента. Пациент добровольно подписал информированное согласие на публикацию персональной медицинской информации в обезличенной форме.

Конфликт интересов. Авторы декларируют отсутствие явных и потенциальных конфликтов интересов, связанных с публикацией статьи.

Участие авторов. Все авторы внесли значимый вклад в проведение исследования и подготовку статьи, прочли и одобрили финальную версию статьи перед публикацией. 


\section{СПИСОК ЛИТЕРАТУРЫ | REFERENCES}

1. Colao A. The prolactinoma. Best Pract Res Clin Endocrinol Metab. 2009;23(5):575-596. doi: 10.1016/j.beem.2009.05.003

2. Gillam MP, Molitch ME, Lombardi G, Colao A. Advances in the Treatment of Prolactinomas. Endocr Rev. 2006;27(5):485-534. doi: 10.1210/er.2005-9998

3. Мельниченко Г.А., Дзеранова Л.К., Пигарова Е.А., и др. Федеральные клинические рекомендации по гиперпролактинемии: клиника, диагностика, дифференциальная диагностика и методы лечения. Проблемы эндокринологии. - 2013. - Т.59. - №6. - С.19-26. [Mel'nichenko GA, Dzeranova LK, Pigarova EA, et al. Russian association of endocrinologists national practice guidelines (clinical signs, diagnosis, differential diagnosis, treatment). Hyperprolactinemia.

Problems of endocrinology. 2013;59(6):19-26. (In Russ.)] doi: 10.14341/probl201359619-26

4. Дзеранова Л.К., Федорова Н.С., Воротникова С.Ю., и др. Описание клинических портретов пациентов С гиперпролактинемией. Ожирение и метаболизм. 2018. - T.15. - №3. - C.65-69. [Dzeranova LK, Fedorova NS, Vorotnikova SY, et al. Description of clinical portraits of patients with hyperprolactinemia // Obesity and Metabolism. 2018;15(3):65-69.

(In Russ.)] doi:10.14341/omet9866

5. Melmed S, Casanueva FF, Hoffman AR, et al. Diagnosis and Treatment of Hyperprolactinemia: An Endocrine Society Clinical Practice Guideline. J Clin Endocrinol Metab. 2011;96(2):273-288

doi: 10.1210/jc.2010-1692

6. Webster J, Piscitelli G, Polll A, et al. The efficacy and tolerability of long-term cabergoline therapy in hyperprolactinaemic disorders: an open, uncontrolled, multicentre study. Clin Endocrinol (Oxf). 1993;39(3):323-329. doi: 10.1111/j.1365-2265.1993.tb02372.x

7. Webster J, Piscitelli G, Polli A, Ferrari Cl, Ismail I, Scanlon MF. A Comparison of Cabergoline and Bromocriptine in the Treatment of Hyperprolactinemic Amenorrhea. N Engl J Med. 1994;331(14):904-909. doi: 10.1056/NEJM199410063311403

8. SABUNCU T, ARIKAN E, TASAN E, HATEMI H. Comparison of the Effects of Cabergoline and Bromocriptine on Prolactin Levels in Hyperprolactinemic Patients. Intern Med. 2001;40(9):857-861.

doi: 10.2169/internalmedicine 40.857

9. Федорова Н.С. Клинико-морфологическая характеристика пролактинсекретирующих опухолей гипофиза, резистентных к лечению агонистами дофамина: Диссертация на соискание ученой степени кандидата медицинских наук - Москва; 2018. [Fedorova NS. Kliniko-morfologicheskaya kharakteristika prolaktinsekretiruyushchikh opukholei gipofiza, rezistentnykh k lecheniyu agonistami dofamina. [dissertation] Moscow; 2018. (In Russ.)] Доступно по: https://www.endocrincentr.ru/sites/ default/files/specialists/science/dissertation/avtoreferat_fedorova_v2.0.pdf

10. Vroonen $L$, Jaffrain-Rea $M-L$, Petrossians $P$, et al. Prolactinomas resistant to standard doses of cabergoline: a multicenter study of 92 patients. Eur J Endocrinol. 2012;167(5):651-662. doi: 10.1530/EJE-12-0236

11. Molitch ME. Management of medically refractory prolactinoma. J Neurooncol. 2014;117(3):421-428. doi: 10.1007/s11060-0131270-8

12. Molitch ME. Pharmacologic Resistance in Prolactinoma Patients. Pituitary. 2005;8(1):43-52. doi: 10.1007/s11102-005-5085-2

13. Cannavò S, Bartolone L, Blandino A, et al. Shrinkage of a PRLsecreting pituitary macroadenoma resistant to cabergoline. J Endocrinol Invest. 1999:22(4):306-309. doi: 10.1007/BF03343561

14. Мельниченко Г.А., Дзеранова Л.К., Бармина И.И., и др. Резистентность ктерапии агонистами дофамина у пациентов с гиперпролактинемией // Вестник репродуктивного здоровья. 2007. - №1. - C.33-41. [Mel'nichenko GA, Dzeranova LK, Barmina II, et al. Rezistentnost'k terapii agonistami dofamina u patsientov s giperprolaktinemiey // Bulletin of Reproductive Health 2007:(1):33-41 (In Russ.)] doi: 10.14341/brh2007133-41
15. Maiter D. Management of Dopamine Agonist-Resistant Prolactinoma. Neuroendocrinology. 2019;109(1):42-50. doi: 10.1159/000495775

16. Kissner DG, Jarrett JC. Side effects of bromocriptine. N Engl J Med. 1980;302(13):749-750. doi: 10.1056/NEJM198003273021313

17. Zanettini R, Antonini A, Gatto G, et al. Valvular Heart Disease and the Use of Dopamine Agonists for Parkinson's Disease. Cohadon F, Dolenc V V, Antunes JL, et al., eds. N Engl J Med. 2007;356(1):39-46. doi: 10.1056/NEJMoa054830

18. Schade R, Andersohn F, Suissa S, et al. Dopamine Agonists and the Risk of Cardiac-Valve Regurgitation. N Engl J Med. 2007;356(1):29-38. doi: 10.1056/NEJMoa062222

19. Molitch ME. Drugs and prolactin. Pituitary. 2008;11(2):209-218. doi: 10.1007/s11102-008-0106-6

20. Bhatt MH, Keenan SP, Fleetham JA, Calne DB. Pleuropulmonary disease associated with dopamine agonist therapy. Ann Neurol. 1991;30(4):613-616. doi: 10.1002/ana.410300416

21. Frans E, Dom R, Demedts M. Pleuropulmonary changes during treatment of Parkinson's disease with a long-acting ergot derivative, cabergoline. Eur Respir J. 1992:5(2):263-265. PMID: 1348483

22. Сапронова М.Р., Шнайдер Н.А. Предикторы и модификаторы импульсивно-Компульсивных расстройств при болезни Паркинсона // Журнал неврологии и психиатрии. - 2016. T.116 - №11. - C.145 2016;116(11):145. [Sapronova MR., Schneider NA. Predictors and modifiers of impulsive compulsive disorders in Parkinson's disease. Zhurnal nevrologii i psikhiatrii imeni S.S. Korsakova. 2016;116(11):145. (In Russ.)] doi: 10.17116/jnevro2016116111145-156

23. Vallelunga A, Flaibani R, Formento-Dojot P, Biundo R, Facchini S, Antonini A. Role of genetic polymorphisms of the dopaminergic system in Parkinson's disease patients with impulse control disorders. Parkinsonism Relat Disord. 2012;18(4):397-399. doi: 10.1016/j.parkreldis.2011.10.019

24. Giladi N, Weitzman N, Schreiber S, Shabtai H, Peretz C. New onset heightened interest or drive for gambling, shopping, eating or sexual activity in patients with Parkinson's disease: the role of dopamine agonist treatment and age at motor symptoms onset. J Psychopharmacol. 2007;21(5):501-506. doi: 10.1177/0269881106073109

25. Проскурина И.А., Романцова Т.И. Новые избирательные стимуляторы дофаминовых рецепторов в лечении гиперпролактинемического гипогонадизма. Международный журнал медицинской практики. - 2001. - №1. - C.36-46. [Proskurina IA, Romantsova TI. Novye izbiratel'nye stimulyatory dofaminovykh retseptorov $v$ lechenii giperprolaktinemicheskogo gipogonadizma. Mezhdunarodnyi zhurnal meditsinskoi praktiki. 2001;(1):36-46 (In Russ.)]

26. Ono M, Miki N, Kawamata T, et al. Prospective Study of High-Dose Cabergoline Treatment of Prolactinomas in 150 Patients. J Clin Endocrinol Metab. 2008;93(12):4721-4727. doi: 10.1210/jc.2007-2758

27. Vilar $L$, Vilar C, Albuquerque $\mathrm{J} L$, et al. The use of increasing doses of cabergoline in the management of cabergoline-resistant prolactinomas. Presented at 19th European Congress of Endocrinology May 2017, Lisbon, Portugal. Endocr Abstr. (2017)49EP975

doi: 10.1530/endoabs.49.EP975

28. Gillam MP, Middler S, Freed DJ, Molitch ME. The Novel Use of Very High Doses of Cabergoline and a Combination of Testosterone and an Aromatase Inhibitor in the Treatment of a Giant Prolactinoma. J Clin Endocrinol Metab. 2002;87(10):4447-4451. doi: 10.1210/jc.2002-020426

29. Delgrange E, Daems T, Verhelst J, et al. Characterization of resistance to the prolactin-lowering effects of cabergoline in macroprolactinomas: a study in 122 patients. Eur J Endocrinol. 2009;160(5):747-752. doi: 10.1530/EJE-09-0012

30. Di Sarno A, Landi ML, Cappabianca P, et al. Resistance to Cabergoline as Compared with Bromocriptine in Hyperprolactinemia: Prevalence, Clinical Definition, and Therapeutic Strategy. J Clin Endocrinol Metab. 2001;86(11):5256-5261. doi: 10.1210/jcem.86.11.8054 
ИНФОРМАЦИЯ ОБ АВТОРАХ [AUTHORS INFO]:

*Калмыкова Зиля Асхатовна, ординатор [Zilya A. Kalmykova, MD, residence];

адрес: Россия, 117036, Москва, улица Дм. Ульянова, д.11 [address: 11 Dmitriya Ulyanova street, 117036 Moscow, Russia]; ORCID: https://orcid.org/0000-0002-2612-5253; eLibrary SPIN: 1264-0320; e-mail: zilya.kalmykova@gmail.com

Воротникова Светлана Юрьевна [Svetlana Y. Vorotnikova, MD];

ORCID: https://orcid.org/0000-0001-7470-1676; eLibrary SPIN: 6571-1206; e-mail: bra_svetix@list.ru

Федорова Наталья Сергеевна, к.M.H. [Natalia S. Fedorova, MD, PhD];

ORCID: https://orcid.org/0000-0002-9816-5043; eLibrary SPIN: 6599-5612; e-mail: fedorova.n.s.12@gmail.com

Дзеранова Лариса Константиновна, д.м.н. [Larisa K. Dzeranova, MD, PhD];

ORCID: https://orcid.org/0000-0002-0327-4619; eLibrary SPIN: 2958-5555; e-mail: dzeranovalk@yandex.ru

Пигарова Екатерина Александровна, К.м.н. [Ekaterina A. Pigarova, MD, PhD];

ORCID: http://orcid.org/0000-0001-6539-466X; eLibrary SPIN: 6912-6331; e-mail: kpigarova@gmail.com

Воронцов Александр Валерьевич, д.м.н., профессор [Aleksandr V. Vorontsov, MD, PhD, professor];

ORCID: http://orcid.org/0000-0002-2791-3278; eLibrary SPIN: 2502-4463; e-mail: mr2005i@mail.ru

\section{ЦИТИРОВАТЬ:}

Калмыкова 3.А., Воротникова С.Ю., Федорова Н.С., Дзеранова Л.К., Пигарова Е.А., Воронцов А.В. Эффективность назначения высоких доз каберголина при лечении резистентных пролактином: клиническое наблюдение // Ожирение и метаболизм. — 2019. — Т.16. — №2. - C.89-94. doi: https://doi.org/10.14341/omet10243

\section{TO CITE THIS ARTICLE:}

Kalmykova ZA, Vorotnikova SY, Fedorova NS, Dzeranova LK, Pigarova EA, Vorontsov AV. The efficacy of high-dose cabergoline treatment of prolactinomas resistant to standard doses: a clinical observation. Obesity and metabolism. 2019;16(2):89-94. doi: https://doi.org/10.14341/omet10243 